\title{
Research Article \\ Urinary Peptides Associated Closely with Gestational Diabetes Mellitus
}

\author{
Zhiying Hu, ${ }^{1,2,3}$ Yaping Tian, ${ }^{3}$ Jia Li, ${ }^{1,2}$ Mei Hu, ${ }^{1,2}$ and Man Zhang $\mathbb{D}^{1,2}$ \\ ${ }^{1}$ Clinical Laboratory Medicine, Beijing Shijitan Hospital, Capital Medical University, Beijing 100038, China \\ ${ }^{2}$ Beijing Key Laboratory of Urinary Cellular Molecular Diagnostics, Beijing 100038, China \\ ${ }^{3}$ Laboratory of Translational Medicine, The First Medical Center of Chinese PLA General Hospital, Beijing 100853, China
}

Correspondence should be addressed to Man Zhang; zhangman@bjsjth.cn

Received 2 April 2020; Revised 3 July 2020; Accepted 5 August 2020; Published 21 August 2020

Academic Editor: Manfredi Rizzo

Copyright @ 2020 Zhiying Hu et al. This is an open access article distributed under the Creative Commons Attribution License, which permits unrestricted use, distribution, and reproduction in any medium, provided the original work is properly cited.

Gestational diabetes mellitus (GDM) is a common disease of pregnant women, which has a higher incidence in recent years. The purpose of this study is to explore urinary biomarkers that could predict and monitor gestational diabetes mellitus (GDM). Urine samples from 30 normal pregnant women and 78 GDM patients were collected and purified by weak cationic exchange magnetic beads (MB-WCX), then analyzed by matrix-assisted laser desorption ionization time of flight mass spectrometry (MALDI-TOFMS). The urinary peptide signatures of the two groups were compared by BioExplorer software. The potential ability of the differently expressed peptides to distinguish GDM patients from normal pregnant women was evaluated by receiver operating characteristic (ROC) analysis. At last, the differently expressed peptides were identified by liquid chromatography tandem mass spectrometry (LC-MS). There were four differently expressed peptides $(\mathrm{m} / \mathrm{z} 1000.5,1117.5,1142.9$, and 2022.9) between two groups, which were identified as fragments of urinary albumin, $\alpha 2$-macroglobulin, human hemopexin, and $\alpha 1$-microglobulin, respectively. The diagnostic efficacy of $m / z 1142.9$ was better than the other peptides. The area under the curve (AUC) of the $m /$ $z 1142.9$ was 0.690 (95\% CI: $0.583-0.796)$. The discovery of urinary polypeptides provides the possibility for the early prediction of GDM and the monitoring of glucose metabolism in GDM patients by a noninvasive method.

\section{Introduction}

Gestational diabetes mellitus (GDM) can increase the rate of miscarriage, lead to fetal growth restriction, fetal malformation, macrosomia, neonatal respiratory distress syndrome, neonatal hypoglycemia, and other adverse prognoses, and significantly increase the probability of type 2 diabetes in mothers and offspring in the long term [1-5]. Several studies show that GDM treatment can reduce the incidence of adverse pregnancy outcomes [6-8]. In order to monitor the glucose metabolism of GDM patients, the fasting plasma glucose (FPG) and glycosylated hemoglobin are currently used in the clinic. FPG detection can realize the real-time monitoring of glucose metabolism, but it needs repeatedly invasive blood collection operations by nurses. The traumatic operation leads to the poor compliance of GDM patients. Although glycosylated hemoglobin can effectively reflect the blood glucose level of GDM patients in the past 1-2 months, irreversible organ damage to pregnant women and fetuses may have occurred.

In recent years, urinary proteomics has developed rapidly. As the end metabolite, urine has many advantages, such as convenient collecting, completely noninvasive, accumulating more protein types, and reflecting more body pathological changes [9]. As the Beijing Key Laboratory of Urinary Cellular Molecular Diagnostics, the research on small molecular polypeptides in urine of patients with type 2 diabetes has achieved preliminary results [10]. Through the study of urine polypeptides combined with plasma glucose in GDM patients, we hope to provide objective test indexes for the primary screening and auxiliary diagnosis of GDM. The discovery of biomarkers in urine also lays a foundation for the study 
TABLE 1: The clinical characteristics of all subjects $(X \pm S)$.

\begin{tabular}{lcccc}
\hline Parameters & N group $(n=30)$ & GDM group $(n=78)$ & GM1 group $(n=45)$ & GM2 group $(n=33)$ \\
\hline Age (year) & $31.83 \pm 3.71$ & $32.88 \pm 4.21$ & $32.38 \pm 4.11$ & $33.58 \pm 4.32$ \\
Prepregnancy BMI & $21.26 \pm 2.52$ & $23.35 \pm 3.45$ & $22.63 \pm 3.61$ & $24.32 \pm 3.01$ \\
Average gestational age & $39.54 \pm 1.08$ & $38.97 \pm 1.95$ & $39.32 \pm 1.18$ & $38.50 \pm 2.61$ \\
Average number of pregnancies & $1.90 \pm 0.99$ & $2.13 \pm 1.21$ & $2.02 \pm 1.03$ & $2.27 \pm 1.42$ \\
Average number of births & $1.40 \pm 0.50$ & $1.41 \pm 0.55$ & $1.38 \pm 0.53$ & $1.45 \pm 0.56$ \\
Alb/Cr $(\mathrm{mg} / \mathrm{g})$ & $<30$ & $<30$ & $<30$ & $<30$ \\
\hline
\end{tabular}

Notes: Alb/Cr represents the ratio of microalbumin to creatinine in urine.

of the pathological mechanism of GDM and provides a possibility for the prediction and dynamic monitoring of glucose metabolism in patients.

\section{Materials and Methods}

2.1. Study Population. Firstly, the ethics committee of Beijing Shijitan Hospital, Capital Medical University, approved the research project (Research Ethics No. (27) 2018). Secondly, the subjects were all female, aged $24-42$ years; 78 subjects were GDM patients in our hospital from April 2018 to August 2019 (GDM group); 30 subjects were normal pregnant women who completed routine obstetric examination in our hospital in the same period ( $\mathrm{N}$ group). The inclusion criteria are the following: (1) The clinical data of GDM patients and normal pregnant women from 8 weeks to 42 days after delivery were complete. (2) One-step oral glucose tolerance test (OGTT) with $75 \mathrm{~g}$ glucose was performed at 24-28 weeks of gestation. (3) The diagnosis of GDM meets the diabetes diagnosis standard of the American Diabetes Association (ADA) in 2011. The exclusion criteria are the following: (1) GDM subjects with impaired glucose tolerance in the past; (2) all subjects with acute or chronic infections, tumors, and cardiovascular diseases; (3) subjects with severe liver and kidney dysfunction; and (4) GDM subjects with complications such as anemia, abnormal thyroid function (hyperthyroidism and hypothyroidism), pregnancy-induced hypertension syndrome (PIH), preeclampsia, and so on.

78 GDM patients were divided into GM1 (FPG $\leq 4.50$ $\mathrm{mmol} / \mathrm{l} \quad(81 \mathrm{mg} / \mathrm{dl}))$ and GM2 $\quad(\mathrm{FPG}>4.50 \mathrm{mmol} / \mathrm{l}$ $(81 \mathrm{mg} / \mathrm{dl}))$ groups according to the fasting plasma glucose (FPG) level in the OGTT. The clinical characteristics of all subjects are compared as shown in Table 1.

2.2. Urine Sample Collection Preparation. All subjects were informed to refrain from heavy physical activity the day before urine collection. The second void morning urine samples were collected. The urine samples of all subjects had no hematuresis or ketosis. The methods including urine sample pretreatment and temporary storage, fractionation of urinary peptides using weak cationic-exchange magnetic beads (Bioyong Technologies Inc, Beijing, China), MALDI-TOFMS AnchorChip spotting (Bioyong Technologies Inc, Beijing, China), and data acquisition were all performed as previously developed by $\mathrm{Hu}$ et al. [11].
2.3. Statistical Analyses. Descriptive patient characteristics were displayed as the mean \pm SD unless otherwise indicated, and calculations were performed using SPSS 17.0. The FPG results measured by OGTT were collected from GDM patients who have no food intake for more than 8 hours in their second trimester. The peak area was used as quantitative standardization. The comparison of the peak area between two groups was performed by $t$-tests (normal distributed data) or Wilcoxon test (abnormal distributed data) using BioExplorer software. Two-tailed $P$ values $<0.05$ were considered significant in all statistical comparisons. ROC curve analysis and AUC calculations were performed directly with SPSS 17.0 software to determine diagnostic efficacy of each single marker. A binary logistic regression model was established to evaluate the multivariate diagnostic value.

2.4. Peptide Sequence. The $20 \mu \mathrm{l}$ prepared sample of each subject was injected once and identified by LC-MS. The HPLC system EASY-nLC1000 (Thermo Fisher) was used for separation. The liquid phase $\mathrm{A}$ was $0.1 \%$ acetonitrile formate solution ( $2 \%$ acetonitrile), and the liquid phase B was $0.1 \%$ acetonitrile formate solution ( $98 \%$ acetonitrile). The C18 chromatographic column (Thermo Fisher) was equilibrated with $100 \%$ A solution. The flow rate was $200 \mathrm{nl} / \mathrm{min}$. Gradient elution profile was as follows: $2 \%$ B-6\% B-22\% B$32 \%$ B-100\% B-100 \% B in 60 minutes. The samples were separated by capillary high-performance liquid chromatography and analyzed by a Q Exactive plus spectrometer (Thermo Scientific).

2.5. Bioinformatics and Identification of Urine Biomarkers. The spectra were analyzed with Peaks8.5 (Bioinformatics Solutions Inc.), and the resulting mass lists were matched against the IPI Human database (v3.45) using Sequest search. Parameters were set as follows: Delton $\geq 0.1$; charge2+, Xcorr2.0; charge3+, Xcorr2.5; peptide probability $\leq 1 e-003$; parent ion mass tolerance: $10 \mathrm{ppm}$; fragment ion mass tolerance: $0.02 \mathrm{Da}$; enzyme: no enzyme; variable modification: oxidation of methionine.

\section{Results}

3.1. Urinary Peptidome Profiling. Urine samples from 108 volunteers were purified by MB-WCX. After analysis of MALDI-TOF-MS, typical WCX spectra are shown in Figure 1. 


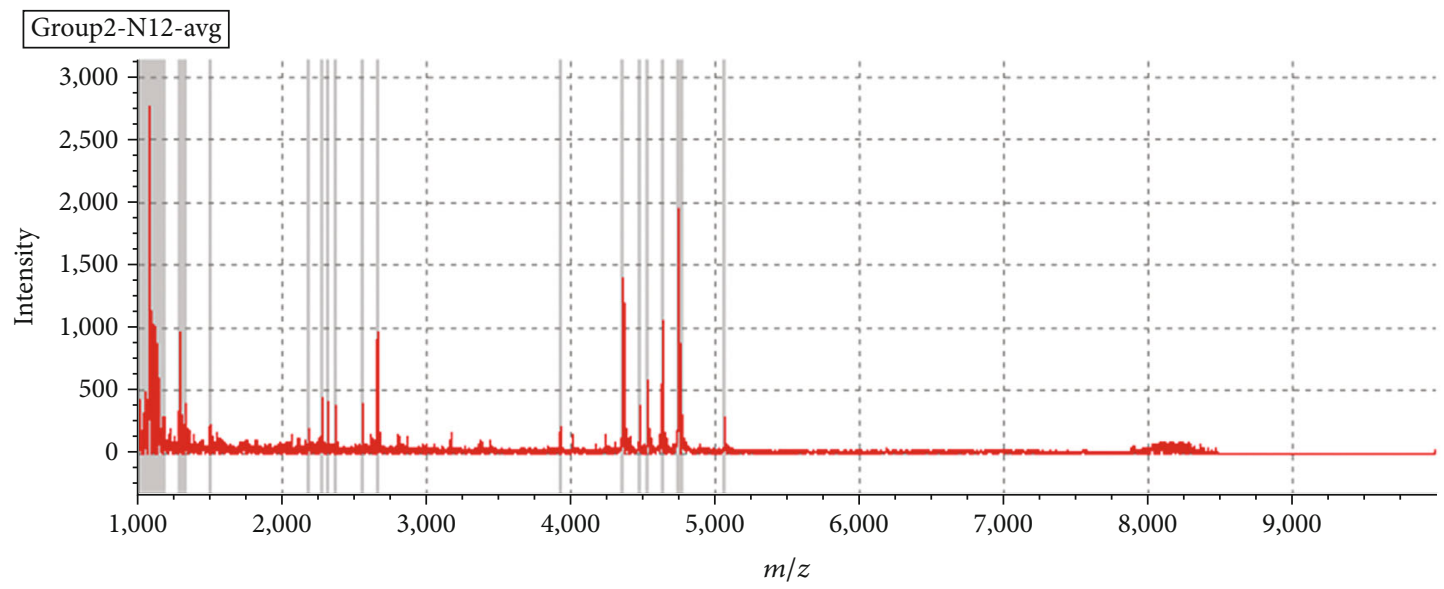

(a)

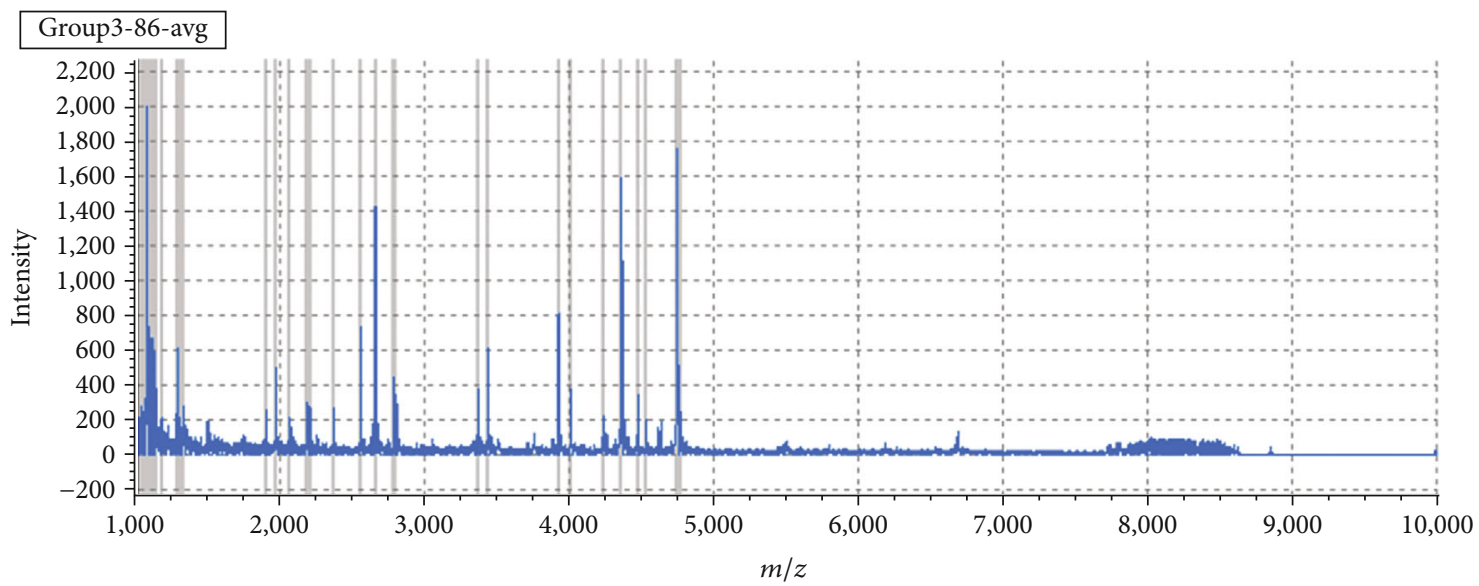

(b)

FIGURE 1: Typical urinary sample mass spectra from MALDI-TOF-MS after being purified by MB-WCX: (a) one sample of a normal pregnant woman; (b) one sample of a GDM patient.

3.2. Statistical Analysis between N Group and GDM Group. Using BioExplorer software, 172 distinguishable peaks were detected within the 1,000 to 10,000 mass charge ratio $(\mathrm{m} / z)$ range; 46 peaks have differential expression and statistical significance $(P<0.05)$ between two groups. We picked eight relatively higher peaks (peak area $>300$ ) for further analysis. The peptides with a mass charge ratio of 1078.6, 1289.6, and 1501.8 had been published [11], and the mass charge ratio of the other five urine peptides was 1000.5, 1117.5, 1142.9, 2022.9, and 4636.5, respectively (Figure 2(a)). Compared to the $\mathrm{N}$ group, $m / z \quad 1000.5$ and 1117.5 were upregulated (Figure 2(b)) and $m / z$ 1142.9, 2022.9, and 4636.5 were downregulated in the GDM group (Figure 2(c)).

3.3. Statistical Analysis between GM1 Group and GM2 Group. The differences of urine polypeptides between the GM1 and GM2 groups with $\mathrm{m} / z$ 1000.5, 1078.6, 1117.5, 1142.9, $1289.6,1501.8,2022.9$, and 4636.5 were analyzed by BioExplorer software. The distribution of $m / z 1000.5,2022.9$, and 4636.5 molecules in two groups was shown in Figure 3(a), and the differences between the two groups were statistically significant $(P<0.05$, Figure 3(b)). Compared with the GM1 group, $\mathrm{m} / \mathrm{z} 1000.5$ and 2022.9 were upregulated and $\mathrm{m} / \mathrm{z}$
4636.5 was downregulated. The distribution of urine polypeptides of $\mathrm{m} / z 1078.6,1117.5,1142.9,1289.6$, and 1501.8 in two groups was shown in Figure 3(c), and there was no significant difference between the two groups $(P>0.05$, Figure 3(d)).

3.4. Trend Analysis. With the increase of FPG in GDM patients, the expression of urine polypeptide with $\mathrm{m} / \mathrm{z}$ 1000.5 increased; the expression of urine polypeptides with $\mathrm{m} / z 2022.9$ and 4636.5 decreased. The trend between the molecule with $\mathrm{m} / z 1000.5$ and FPG was better than that of the other two molecules (Figure 4).

3.5. ROC Analysis. To evaluate the diagnostic efficacy of these peptides, the ROC analysis was performed to calculate the sensitivities, specificities, and accuracies at different cutoff points for differentiating GDM patients from normal pregnant women (Figure 5). In the ROC curves, the AUC of the peptides with $m / z 1000.5,1117.5,1142.9,2022.9$, and 4636.5 were 0.641 (95\% CI: $0.532-0.750), 0.612$ (95\% CI: 0.497-0.726), 0.690 (95\% CI: $0.583-0.796), 0.600$ (95\% CI: $0.476-0.724)$, and 0.759 (95\% CI: 0.655-0.863), respectively. 


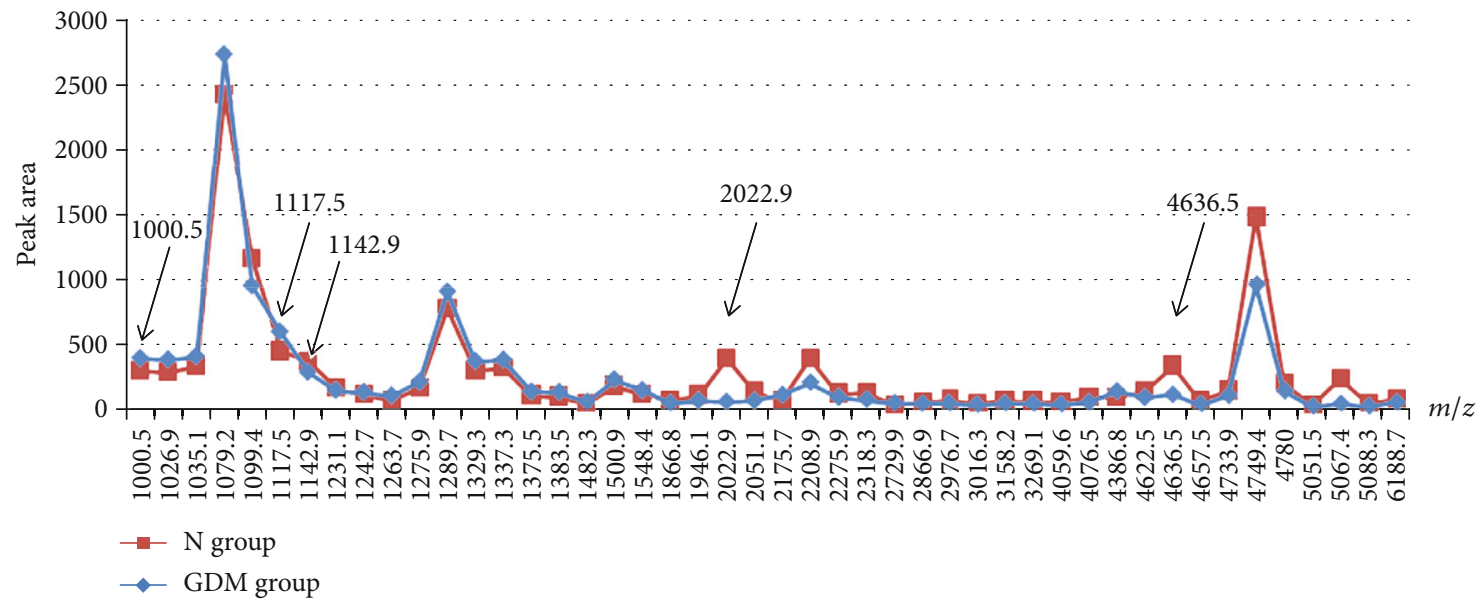

(a)
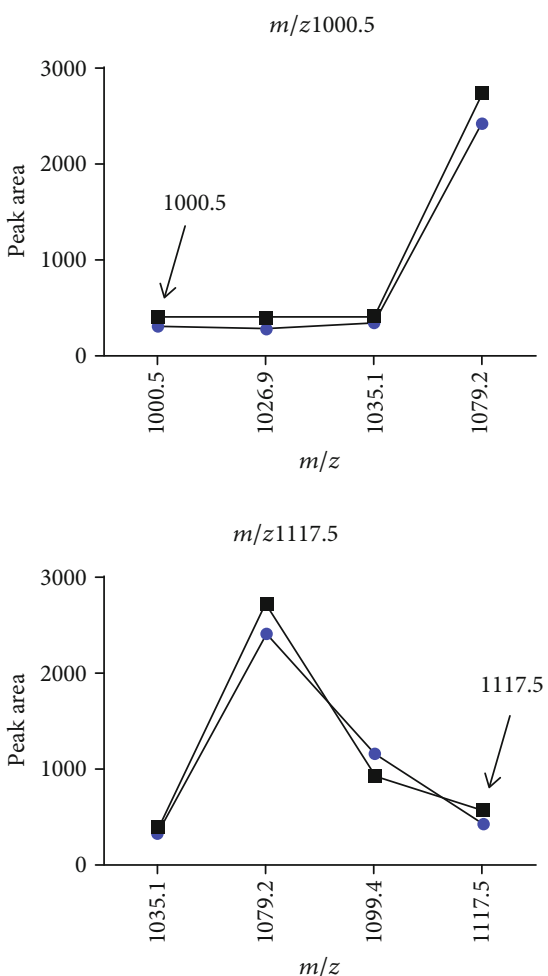

$\rightarrow-\mathrm{N}$ group

$\rightarrow$ GDM group

$m / z 1000.5$
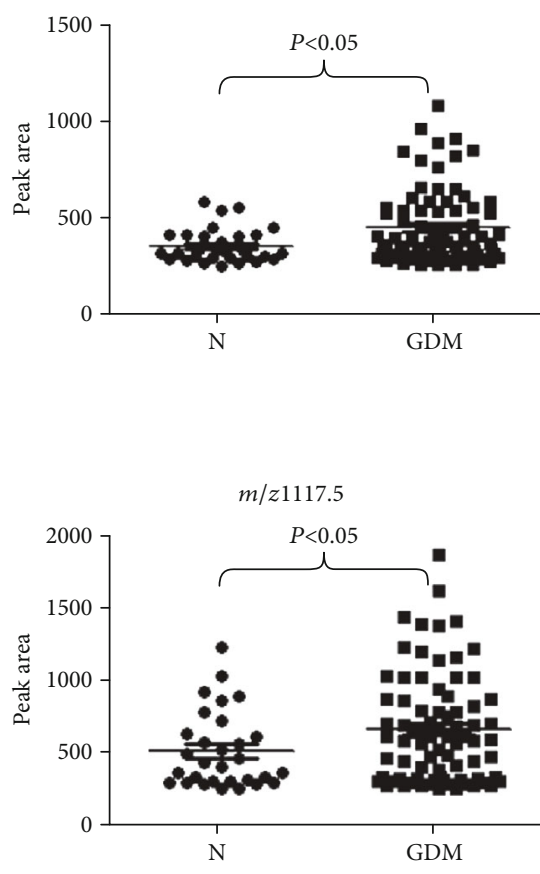

(b)

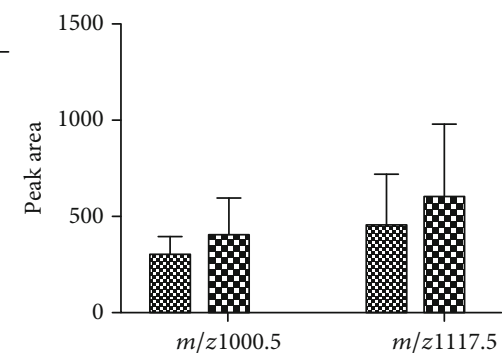

\% $N$ group

$\$$ GDM group

Figure 2: Continued. 

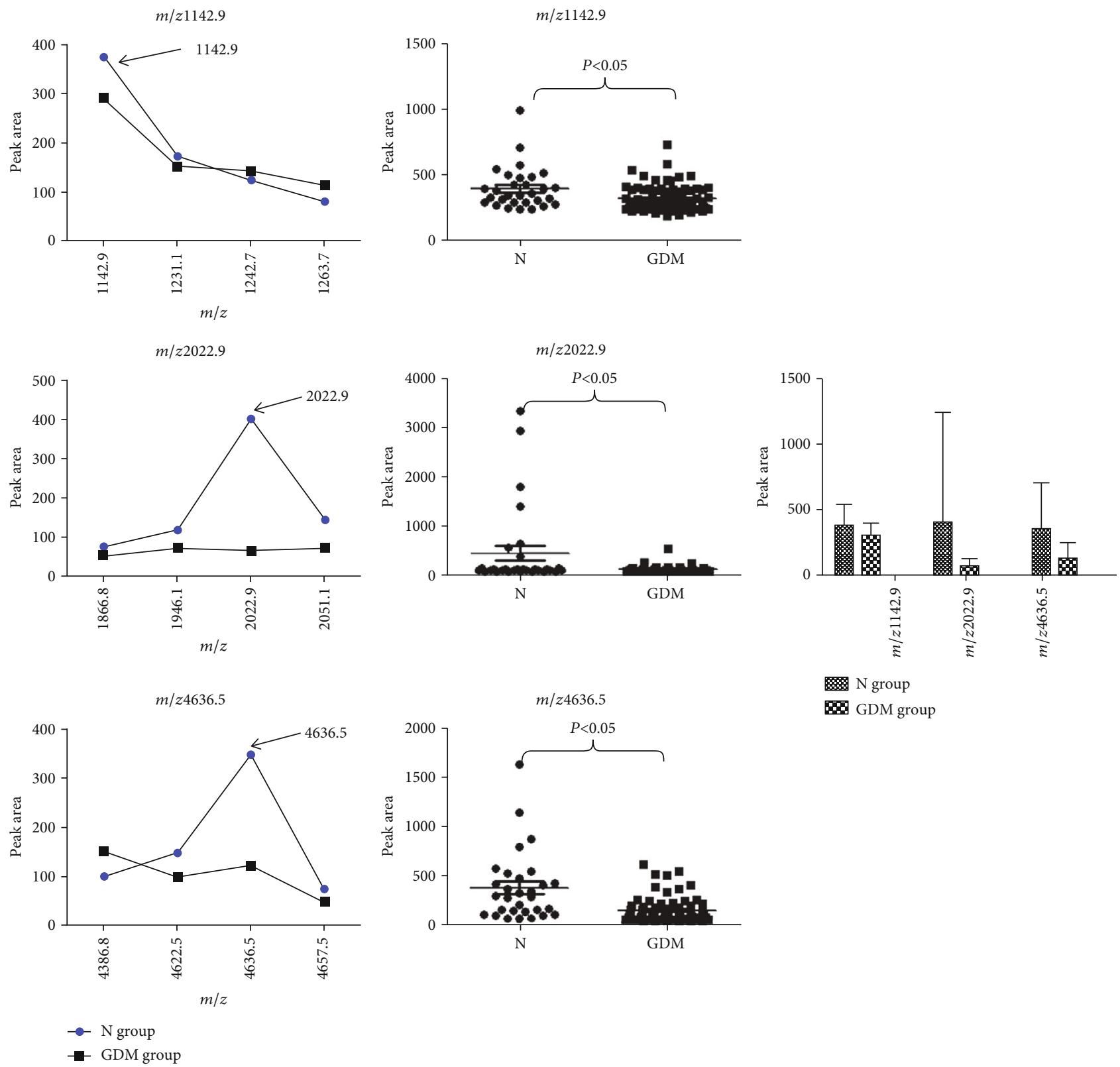

ॠ N group

$\$$ GDM group

(c)

Figure 2: Urine polypeptides in the $\mathrm{N}$ group and the GDM group were analyzed. The samples in the $\mathrm{N}$ and GDM groups were urine samples of 30 normal pregnant women and 78 GDM patients, respectively. (a) The average peak area distributions of all polypeptide peaks were measured in two groups. Five urine polypeptides which were indicated by the arrow were statistically significant in the comparative analysis of two groups $(P<0.05)$. (b) In the comparative analysis of five polypeptides between the N and GDM groups, there were significant differences in the $\mathrm{m} / z 1000.5$ and 1117.5 (left, $P<0.05$ ). The distributions of two polypeptides in two groups are shown (middle). The peak value of two polypeptides in the GDM group was higher than that in the N group (right, $P<0.05$ ). (c) There were significant differences in the $\mathrm{m} / z$ 1142.9, 2022.9, and 4636.5 (left, $P<0.05$ ). The distributions of three polypeptides in two groups are shown (middle). The peak value of three polypeptides in the GDM group was lower than that in the $\mathrm{N}$ group (right, $P<0.05$ ).

In the five indicators, polypeptide with $\mathrm{m} / \mathrm{z} 4636.5 \mathrm{had}$ the best diagnostic value for GDM, with a cutoff value of 235; its sensitivity and specificity were $89.7 \%$ and $56.7 \%$, respectively. Multivariate logistic regression analyses including FPG and polypeptides with $\mathrm{m} / \mathrm{z} 1000.5,1117.5$, $1142.9,2022.9$, and 4636.5 were used to evaluate their diagnostic values. The diagnostic formula was

$$
\begin{aligned}
Y= & \operatorname{logit}(P)=-5.393+1.710 X_{\mathrm{FPG}}+0.007 X_{1000.5} \\
& +0.001 X_{1117.5}-0.007 X_{1142.9}-0.009 X_{2022.9}-0.004 X_{4636.5} .
\end{aligned}
$$

The AUC of multivariate logistic regression was 0.885 (95\% CI: $0.817-0.952$ ). At the cutoff value of 0.774 , the 


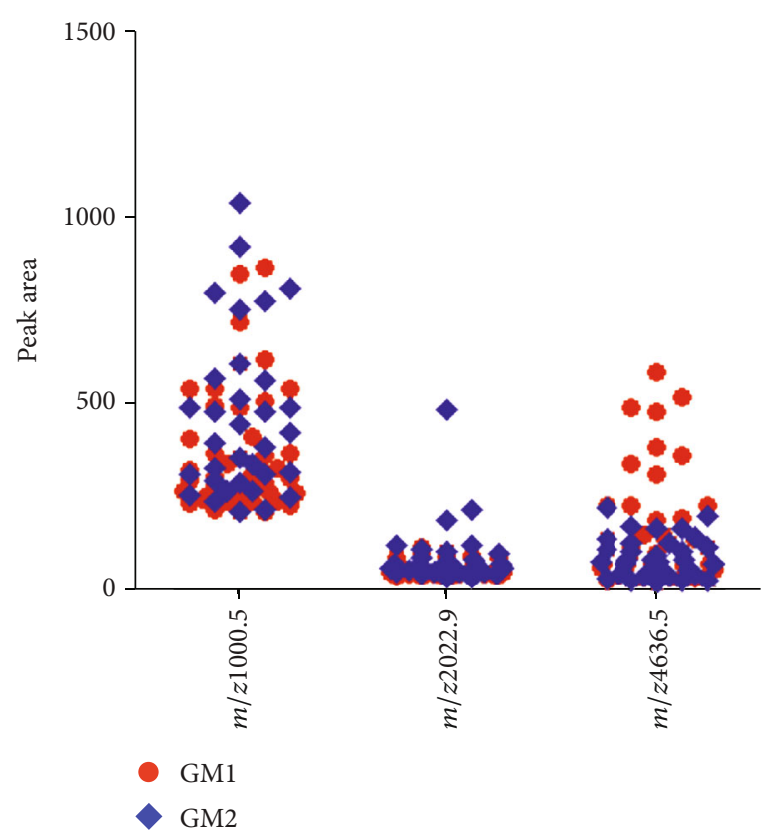

(a)

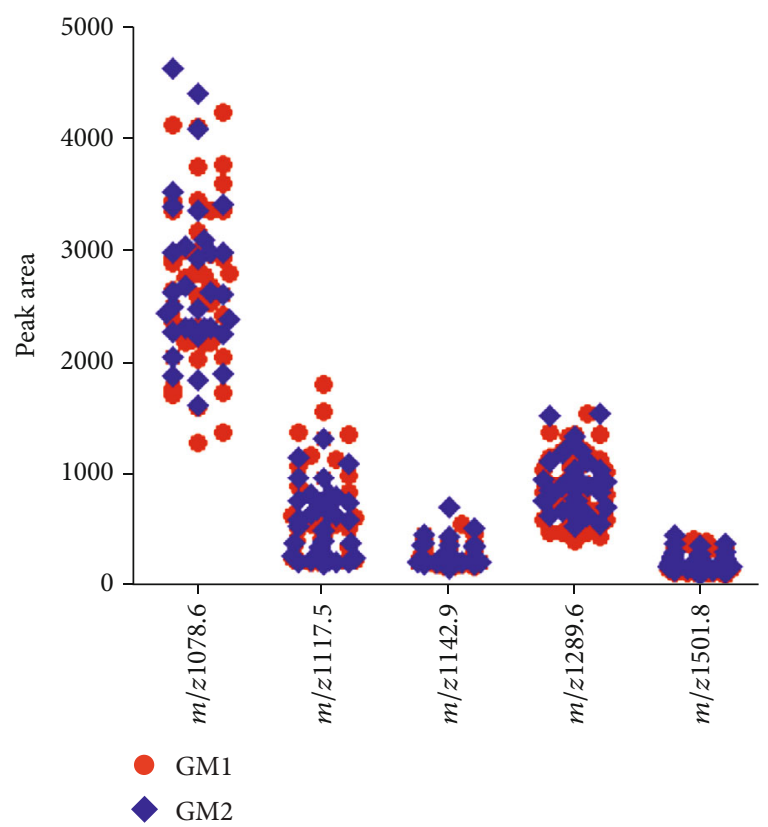

(c)

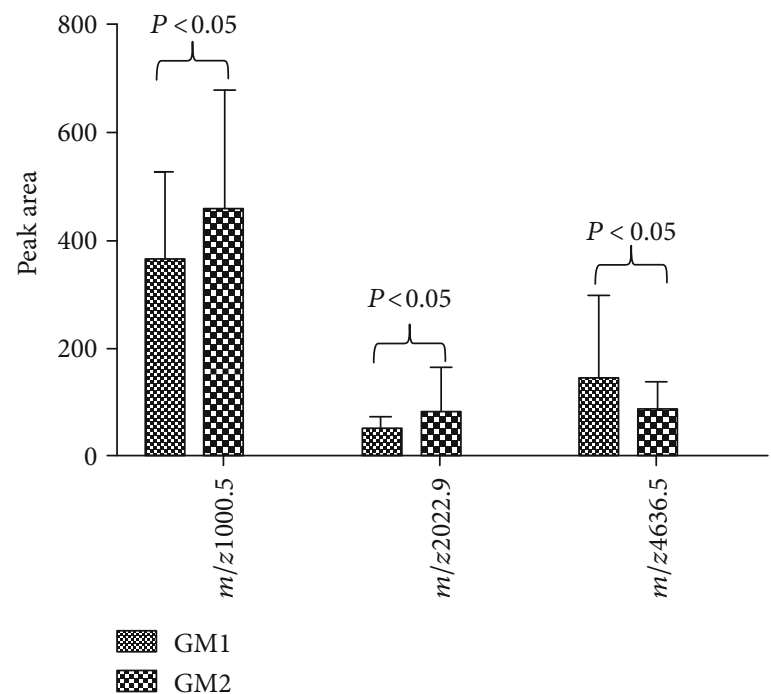

(b)

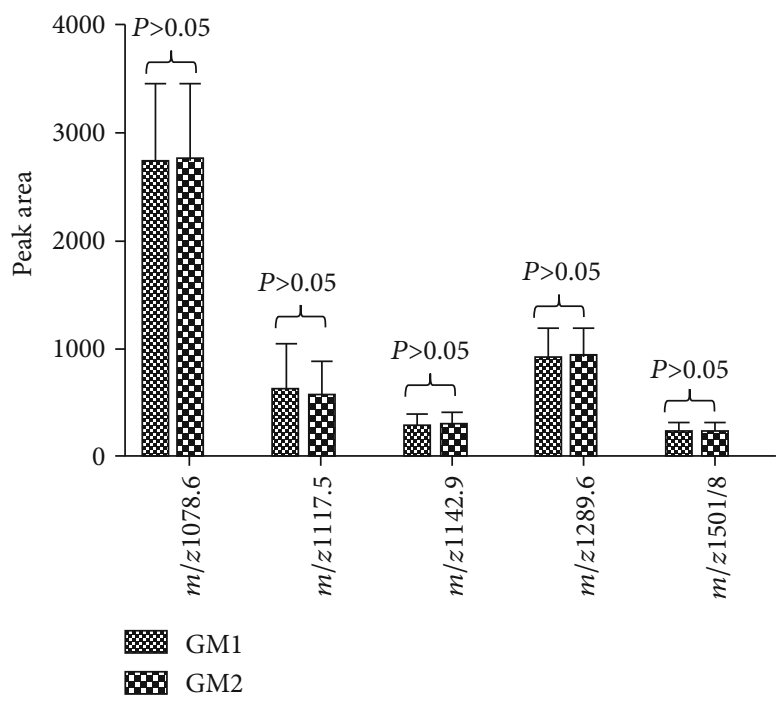

(d)

Figure 3: Comparison of the difference of urine peptides between the GM1 $(n=45)$ and GM2 $(n=33)$ groups. (a) The distribution of molecules with $m / z$ 1000.5, 2022.9, and 4636.5 in two groups is shown. (b) There were significant differences between the GM1 and GM2 groups in three molecules with $\mathrm{m} / z 1000.5,2022.9$, and $4636.5(P<0.05)$. (c) The distribution of molecules with $\mathrm{m} / z 1078.6,1117.5$, $1142.9,1289.6$, and 1501.8 in two groups is shown. (d) There was no significant difference between the GM1 and GM2 groups in three molecules with $\mathrm{m} / \mathrm{z} 1078.6,1117.5,1142.9,1289.6$, and $1501.8(P>0.05)$.

sensitivity and specificity were $83.3 \%$ and $75.6 \%$, respectively.

3.6. Identification of the GDM Potential Urinary Biomarkers. Four molecules with $\mathrm{m} / z$ 1000.5, 1117.5, 1142.9, and 2022.9 were analyzed by LC-MS, and their amino acid sequences were QTALVELVK, QTVSWAVTPK, DYFMPC (+57.02)
PGR, and VVAQGVGIPEDSIFTM (+15.99) ADR. The corresponding names of four molecules were urine albumin (ALBU) precursor, alpha2-macroglobulin (A2MG) precursor, human hemopexin (HEMO) precursor, and alpha1microglobulin (AMBP) precursor by database search. Unfortunately, the identification of the molecule with $\mathrm{m} / \mathrm{z} 4636.5$ failed. The detailed results are shown in Table 2. 


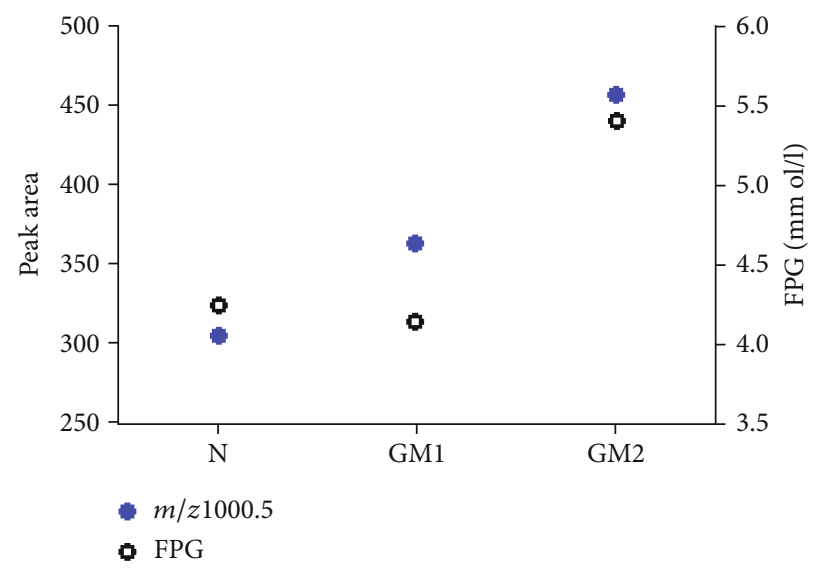

(a)
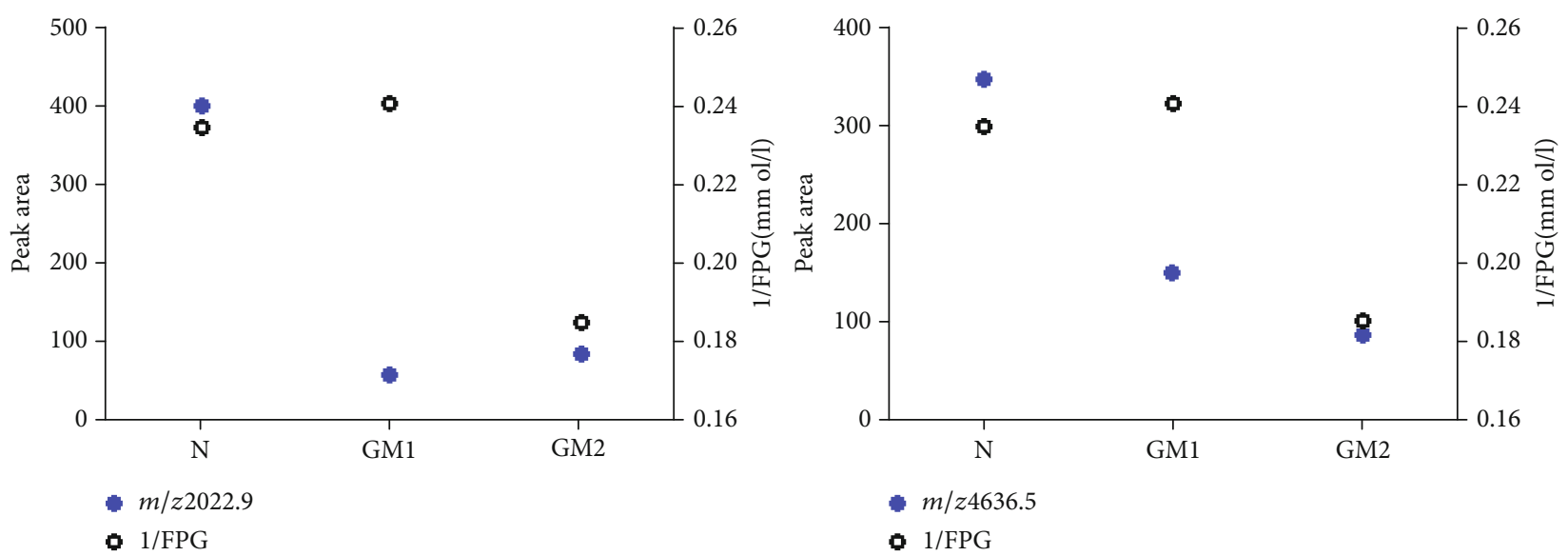

(b)

Figure 4: Trend analysis of differential polypeptide peaks and FPG. (a) Trends of FPG and the molecule with $m / z 1000.5$ in the N group $(n=30)$, GM1 group $(n=45)$, and GM2 group $(n=33)$. (b) Trends of $1 /$ FPG and the molecules with $m / z 2022.0$ and 4636.5 in three groups.

\section{Discussion}

During pregnancy, progressive insulin resistance begins in the second trimester and develops further in the third trimester. Hormones and adipokines secrete by the placenta may be the cause of insulin resistance during pregnancy. Insulin sensitivity begins to decline gradually in the second trimester and becomes more serious in the third trimester [12]. In addition, the increase of estrogen, progesterone, and cortisol during pregnancy helps to destroy the glucose insulin balance [13]. In order to adapt to the insulin resistance and the reduction of sensitivity during pregnancy, insulin secretion increases. GDM occurs when the pancreas does not produce enough insulin to maintain metabolic pressure. GDM can cause a variety of obstetric complications such as hypertension, preeclampsia, premature rupture of membranes, and premature delivery [14-17]. Early prediction of GDM and monitoring of the patients' glucose metabolism level are very important for maternal and infant health.

Urinary proteome is the direction of disease diagnosis, treatment, monitoring, and prognosis research $[9,18]$, and its application fields include urogenital system and other system diseases [19-22]. Comparison of protein patterns in bio- logical fluids between healthy individuals and patients with disease is increasingly being used both to discover biological markers of disease (biomarkers) and to identify biochemical processes important in disease pathogenesis [23]. As the Beijing Key Laboratory of Urinary Cellular Molecular Diagnostics, by analyzing the differences of urinary polypeptide peaks between GDM patients and normal pregnant women, we hope to fully tap the biomarkers of urinary polypeptides in GDM. We grouped GDM patients according to the FPG of OGTT and analyzed the correlation between different polypeptides and disease severity. Four peptides with significant difference were screened out and identified successfully. The protein names are urine albumin (ALBU), alpha2macroglobulin (A2MG), human hemopexin (HEMO), and alpha1-microglobulin (AMBP).

The content of urine microalbumin (UmAlb) in the first trimester is less than $20 \mathrm{mg} / 24 \mathrm{~h}$. As pregnancy continues, UmAlb may slightly increase, but it can remain in the normal range [24]. In the second and third trimesters, the sensitivity of insulin decreases and the demand for insulin increases. GDM patients cannot compensate for the physiological change; then, their plasma glucose rise. The disease results in hypoxia of tissues, increase of blood viscosity, vascular 

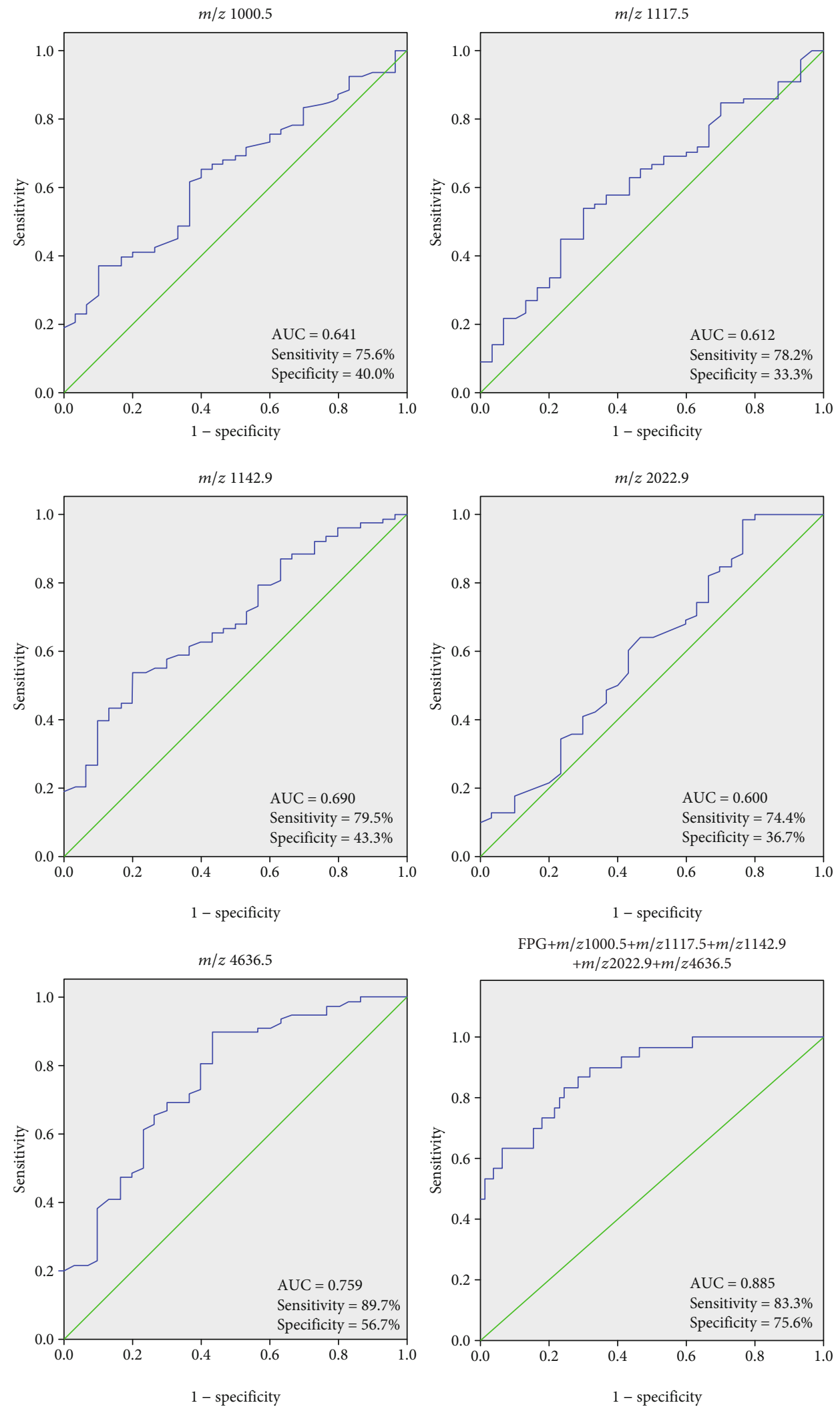

FIGURE 5: ROC analysis. The ROC curve and the AUC were established according to the peak value of urine polypeptides of GDM patients $(n=78)$ and normal pregnant women $(n=30)$ with $m / z 1000.5,1117.5,1142.9,2022.9$, and 4636.5 and comprehensive index. 
TABLE 2: Identification of the GDM potential urinary biomarkers.

\begin{tabular}{lccc}
\hline$m / z$ & Molecular weight & Amino sequence & Protein name \\
\hline 1000.5 & 999.6 & QTALVELVK & Urine albumin precursor \\
1117.5 & 1115.6 & QTVSWAVTPK & Alpha2-macroglobulin precursor \\
1142.9 & 1141.5 & DYFMPC $(+57.02)$ PGR & Human hemopexin precursor \\
2022.9 & 2020.0 & VVAQGVGIPEDSIFTM (+15.99) ADR & Human alpha1-microglobulin precursor \\
4636.5 & & Identification failure & \\
\hline
\end{tabular}

disease, increase of permeability of glomerular basement membrane, and glomerular damage mainly caused by microvascular disease [25]. Several studies show that the detection of UmAlb is a sensitive index for the diagnosis of early renal injury, and it is also the earliest clinical manifestation of diabetic renal microvascular damage [26-29]. GDM-related research points out that the UmAlb level of GDM patients is higher than that of normal pregnant women. Moreover, GDM patients who did not meet the standard of blood glucose control excreted more urine microalbumin. It is suggested that the increase of UmAlb excretion is one of the reasons for GDM progression [24]. In this study, the ratio of microalbumin to creatinine in urine of all subjects was less than $30 \mathrm{mg} / \mathrm{g}$. However, through our urine proteomics study, we can detect the subtle change of UmAlb, and this change is enough to distinguish GDM patients from normal pregnant women. After grouping GDM patients according to FPG, the urinary polypeptide expression in the GM2 group was significantly higher than that in the GM1 group $(P<0.05)$. It indicated that the UmAlb expression increased with the increase of FPG in GDM patients.

Alpha2-macroglobulin (A2MG) is synthesized by hepatocyte and monocyte macrophage system, which is the largest protein in plasma. It is a major plasma protease inhibitor that also regulates the activity of a variety of bioactive peptides including interleukins and exerts a range of immunomodulatory effects [30]. In normal circumstances, A2MG cannot be filtered by the glomerulus, and its content in urine is very little. When the glomerular basement membrane is seriously damaged or blood components enter the urine, the A2MG level in urine increases [31]. According to diabetes researches, A2MG levels were significantly raised in the diabetes type I group. In the group of diabetes type II, A2MG levels are within the normal range. After division of diabetics according to the presence of diabetic complications, A2MG levels in patients with diabetic complications were significantly higher than in the group of diabetics without complications $[32,33]$. The increase in plasma A2MG levels in diabetes may be a correlative measure to encounter the potential proteolytic challenge associated with diabetic microangiopathy, even very early in the course of the disease. A2MG may yet be one of the most specific markers of microvascular complications in diabetes than any other serum protein [30]. At present, there is no report about urine A2MG in GDM. This study showed that the expression of urine A2MG in GDM patients was increased compared with that of normal pregnant women, which was mainly related to glomerular microvascular lesions and increased basement membrane permeability. However, there was no significant difference between GM1 and GM2. Therefore, microexpression of urine A2MG of GDM patients may not be suitable as a marker of glomerular membrane damage.

Human hemopexin (HEMO) is a plasma betaglycoprotein that specifically binds one heme with high affinity and transports it to hepatocytes for salvage of the iron [34]. Some researchers point out that hemopexin is upregulated in plasma from type 1 diabetes mellitus patients due to the effect of glucose-induced reactive oxygen species [35]. There is no report on the relationship between urinary HEMO and GDM. The increase of plasma in normal pregnancy was more than the increase of blood cells, resulting in the dilution of blood and relative anemia. This study found that the expression of urine HEMO in GDM patients was lower than that of normal pregnant women. We consider that although there is glucose-induced reactive oxygen species in GDM patients, the increase of plasma osmolality and blood volume is more obvious, so the final manifestation is the decrease of HEMO expression. The expression of HEMO in the GM2 group was higher than that in the GM1 group, but the difference was not significant.

Urinary AMBP provides a noninvasive and cheap diagnostic method for the diagnosis and monitoring of urinary tract diseases, which can detect renal tubular diseases of diabetic nephropathy in the early stage [36]. GDM-related studies indicated that the urinary AMBP in the GDM group was significantly higher than that in normal pregnant women and normal nonpregnant women. The combined detection of urinary microalbumin and AMBP can early detect the GDM nephropathy, which is a sensitive and effective index for early renal damage monitoring of GDM nephropathy [37]. In this study, compared with normal pregnant women, the urinary AMBP of GDM patients was significantly lower. When the renal tubular reabsorption function is normal, the excretion of urine AMBP will be less than that in normal pregnant women. However, the secretion of AMBP in the GM2 group was significantly higher than that in the GM1 group. It is suggested that with the increase of FPG, renal hypoxia and ischemia aggravated, resulting in glomerular filtration and renal tubular reabsorption dysfunction. With the further increase of blood volume in the third trimester, the secretion of AMBP in GDM patients was lower than that in the second trimester.

Through the study of urinary proteomics of GDM, we further explored the disease-related small urine polypeptides. Combined with FPG, the research results are expected to serve as the basis for the study of the urine glucose metabolism level detection kit. The study also provides early prediction, noninvasive diagnosis, treatment guidance, and prognosis information for GDM patients. 


\section{Data Availability}

The necessary data of this manuscript has been shown in the article.

\section{Ethical Approval}

The ethics committee of Beijing Shijitan Hospital, Capital Medical University, approved the research project. The study was carried out in accordance with the principles of the Declaration of Helsinki and Good Clinical Practice Guidelines.

\section{Conflicts of Interest}

The authors declare that they have no conflict of interest.

\section{Acknowledgments}

Thanks are due to all the volunteers for their generous donation of urine samples. This work was supported by the National Key R\&D Program of China (Nos. 2016YFC1000700 and 2016YFC1000702).

\section{References}

[1] J. P. Vogel, J. P. Souza, R. Mori et al., "Maternal complications and perinatal mortality: findings of the World Health Organization multicountry survey on maternal and newborn health," BJOG: An International Journal of Obstetrics \& Gynaecology, vol. 121, pp. 76-88, 2014.

[2] M. Hod, A. Kapur, D. A. Sacks et al., "The International Federation of Gynecology and Obstetrics (FIGO) Initiative on gestational diabetes mellitus: a pragmatic guide for diagnosis, management, and care," International Journal of Gynecology \& Obstetrics, vol. 131, pp. S173-S211, 2015.

[3] M. Umesawa and G. Kobashi, "Epidemiology of hypertensive disorders in pregnancy: prevalence, risk factors, predictors and prognosis," Hypertension Research, vol. 40, no. 3, pp. 213-220, 2017.

[4] K. Kc, S. Shakya, and H. Zhang, "Gestational diabetes mellitus and macrosomia: a literature review," Annals of Nutrition \& Metabolism, vol. 66, no. 2, Supplement 2, pp. 14-20, 2015.

[5] C. J. Gao, X. M. Huang, Z. P. Chen et al., "High level of hemoglobin during the first trimester of pregnancy associated with the risk of gestational diabetes mellitus," Chinese Journal of Obstetrics and Gynecology, vol. 54, no. 10, pp. 654-659, 2019.

[6] C. A. Crowther, J. E. Hiller, J. R. Moss, A. J. McPhee, W. S. Jeffries, and J. S. Robinson, "Effect of treatment of gestational diabetes mellitus on pregnancy outcomes," The New England Journal of Medicine, vol. 352, no. 24, pp. 2477-2486, 2005.

[7] M. B. Landon, C. Y. Spong, E. Thom et al., "A multicenter, randomized trial of treatment for mild gestational diabetes," The New England Journal of Medicine, vol. 361, no. 14, pp. 13391348, 2009.

[8] E. Cosson, E. Vicaut, D. Sandre-Banon et al., "Initially untreated fasting hyperglycaemia in early pregnancy: prognosis according to occurrence of gestational diabetes mellitus after 22 weeks' gestation: a case-control study," Diabetic Medicine, vol. 37, no. 1, pp. 123-130, 2019.
[9] C. Shao, Y. Wang, and Y. H. Gao, "Applications of urinary proteomics in biomarker discovery (in Chinese)," Science China Life Sciences, vol. 40, pp. 795-804, 2011.

[10] M. Zhang, G. Fu, and T. Lei, "Two urinary peptides associated closely with type 2 diabetes mellitus," PLoS One, vol. 10, no. 4, article e0122950, 2015eCollection 2015.

[11] Z. Hu, M. Zhang, and Y. Tian, "Screening and analysis of small molecular peptides in urine of gestational diabetes mellitus," Clinica Chimica Acta, vol. 502, pp. 174-182, 2020.

[12] J. Drobny, "Metabolic syndrome and the risk of preeclampsia," Bratislavské Lekárske Listy, vol. 110, no. 7, pp. 401-403, 2009.

[13] Y. Sun, H. Yang, and W. J. Sun, "Risk factors for pre-eclampsia in pregnant Chinese women with abnormal glucose metabolism," International Journal of Gynecology \& Obstetrics, vol. 101, no. 1, pp. 74-76, 2008.

[14] The HAPO Study Cooperative Research Group, "Hyperglycemia and adverse pregnancy outcomes," New England Journal of Medicine, vol. 358, pp. 1991-2002, 2008.

[15] D. M. Jensen, B. Sorensen, N. Feilberg-Jorgensen, J. G. Westergaard, and H. Beck-Nielsen, "Maternal and perinatal outcomes in 143 Danish women with gestational diabetes mellitus and 143 controls with a similar risk profile," Diabetic Medicine, vol. 17, no. 4, pp. 281-286, 2000.

[16] N. M. Nordin, J. W. H. Wei, N. N. Naing, and E. M. Symonds, "Comparison of maternal-fetal outcomes in gestational diabetes and lesser degrees of glucose intolerance+," Journal of Obstetrics and Gynaecology Research, vol. 32, no. 1, pp. 107114, 2006.

[17] C. J. Magri, N. Calleja, G. Buhagiar, S. Fava, and J. Vassallo, "Factors associated with diabetic nephropathy in subjects with proliferative retinopathy," International Urology and Nephrology, vol. 44, no. 1, pp. 197-206, 2012, Epub 2011 Apr 24.

[18] S. Decramer, A. G. de Peredo, B. Breuil et al., "Urine in clinical proteomics," Molecular and Cellular Proteomics, vol. 7, no. 10, pp. 1850-1862, 2008.

[19] L. Chu, G. Fu, Q. Meng, H. Zhou, and M. Zhang, "Identification of urinary biomarkers for type 2 diabetes using beadbased proteomic approach," Diabetes Research and Clinical Practice, vol. 101, no. 2, pp. 187-193, 2013, Epub 2013 Jun 13.

[20] T. Lei, X. Zhao, S. Jin, Q. Meng, H. Zhou, and M. Zhang, "Discovery of potential bladder cancer biomarkers by comparative urine proteomics and analysis," Clinical Genitourinary Cancer, vol. 11, no. 1, pp. 56-62, 2013.

[21] J. Chen, L. J. Chen, Y. L. Xia et al., "Identification and verification of transthyretin as a potential biomarker for pancreatic ductal adenocarcinoma," Journal of Cancer Research and Clinical Oncology, vol. 139, no. 7, pp. 1117-1127, 2013.

[22] D. Xiao, F. L. Meng, L. H. He, Y. X. Gu, and J. Z. Zhang, “Analysis of the urinary peptidome associated with Helicobacter pylori infection," World Journal of Gastroenterology, vol. 17, no. 5, pp. 618-624, 2011.

[23] J. Barratt and P. Topham, "Urine proteomics: the present and future of measuring urinary protein components in disease," Canadian Medical Association Journal, vol. 177, no. 4, pp. 361-368, 2007.

[24] H. Wang, L. Rong, and W. Guo, "Levels of serum homocysteine and urinary microalbumin in patients with gestational diabetes mellitus and their correlation with pregnancy outcome (in Chinese)," Hainan Medical Journal, vol. 30, no. 7, 2019. 
[25] R. Xu, "Recent progress of biomarkers for diabetic kidney disease (in Chinese)," Chinese Journal of Laboratory Medicine, vol. 38, no. 9, pp. 583-585, 2015.

[26] M. Rodrigues and C. B. Dias, "Microalbuminuria in non diabetic population as an marker of nephropathy," Jornal Brasileiro de Nefrologia, vol. 38, no. 2, pp. 203-208, 2016.

[27] S. Isobe, M. Yuba, H. Mori et al., "Increased pre-procedural urinary microalbumin is associated with a risk for renal functional deterioration after coronary computed tomography angiography," International Journal of Cardiology, vol. 230, pp. 599-603, 2017.

[28] U. G. Thakkar, H. L. Trivedi, A. V. Vanikar, and S. D. Dave, "Co-infusion of insulin-secreting adipose tissue-derived mesenchymal stem cells and hematopoietic stem cells: novel approach to management of type 1 diabetes mellitus," International Journal of Diabetes in Developing Countries, vol. 36, no. 4, pp. 426-432, 2016.

[29] N. Melamed, L. Hiersch, M. Hod, R. Chen, A. Wiznitzer, and Y. Yogev, "Is abnormal 50g glucose challenge testing an independent predictor of adverse pregnancy outcome?," The Journal of Maternal-Fetal \& Neonatal Medicine, vol. 25, no. 12, pp. 2583-2587, 2012.

[30] J. Ahmad, M. Singh, and M. Saleemuddin, "A study of plasma alpha-2-macroglobulin levels in type 2 diabetic subjects with microalbuminuria," The Journal of the Association of Physicians of India, vol. 49, pp. 1062-1065, 2001.

[31] T. Narita, H. Kitazato, J. Koshimura, K. Suzuki, M. Murata, and S. Ito, "Effects of protein meals on the urinary excretion of various plasma proteins in healthy subjects," Nephron, vol. 81, no. 4, pp. 398-405, 1999.

[32] L. Turecký, V. Kupcová, and M. Szántová, "Alpha 2macroglobulin in the blood of patients with diabetes mellitus," Bratislavské Lekárske Listy, vol. 100, no. 1, pp. 25-27, 1999.

[33] V. do Nascimento de Oliveira, A. B. M. L. Neto, M. F. van Tilburg et al., "Proteomic analysis to identify candidate biomarkers associated with type 1 diabetes," Diabetes, Metabolic Syndrome and Obesity: Targets and Therapy, vol. 11, pp. 289-301, 2018.

[34] N. Takahashi, Y. Takahashi, and F. W. Putnam, "Complete amino acid sequence of human hemopexin, the hemebinding protein of serum," Proceedings of the National Academy of Sciences, vol. 82, no. 1, pp. 73-77, 1985.

[35] C. C. Chen, Y. C. Lu, Y. W. Chen et al., "Hemopexin is upregulated in plasma from type 1 diabetes mellitus patients: role of glucose-induced ROS," Journal of Proteomics, vol. 75, no. 12, pp. 3760-3777, 2012, Epub 2012 May 8.

[36] N. Shore, R. Khurshid, and M. Saleem, "Alpha-1 microglobulin: a marker for early detection of tubular disorders in diabetic nephropathy," Journal of Ayub Medical College, Abbottabad, vol. 22 , no. 4 , pp. 53-55, 2010.

[37] S. Li, X. Lu, and J. Zou, "Application value of joint test on urine albumin, $\alpha 1$-micro globulin and transferrin in early renal damage result from the gestational diabetes (in Chinese)," China Medicine and Pharmacy, vol. 1, no. 21, pp. 32-33, 2011. 